\title{
Ethnobotany of Weeds; Weed Flora of Ramat Polytechnic Teaching and Research Farm, Maiduguri Borno State of Nigeria
}

\author{
Y. B. Kajidu ${ }^{1}$, Adam B. K ${ }^{1}$, R. A. Saleh ${ }^{2}$, I. B. Shehu ${ }^{3}$ and Y. M. Kundili ${ }^{3}$ \\ ${ }^{1}$ Department of Crop Production, Faculty of Agriculture, University of Maiduguri \\ ${ }^{2}$ Desert Research Monitoring and Control Centre, Yobe State University, Nigeria \\ ${ }^{3}$ Department of Agricultural Technology Ramat Polytechnic \\ Maiduguri Borno State. Nigeria
}

\begin{abstract}
This study was carried out to ascertain the species of weeds at Ramat Polytechnic Teaching and Research Farm, Maiduguri Borno State Nigeria. The objective of the study is to identify the weeds flora and ethnobotanical benefits of these weeds. Weed flora shows that 38 weed species were identified belonging to 18 different families out of which mostly, twenty-six (26) are annuals the remaining (8) are perennials. The distribution of these weeds further shows that Cleome gynandra L., Commelina benghalens is Schult. F., Euphorbia hirta linn., Crotalaria mucronata L., Senna occidentalis L., Boerhavia erecta L., Eragrotis tenella (A. Rich) Hoschst. Ex Steud., Cenchrus biflorus (Roxb.)., Corchorus olitorius L. and Tribulus terrestris L. have high (70\%) occurrence at the farm. The dominant plant families are the Fabaceae, Poaceae and Malvaceae. Most of the samples weeds have uses ranging from medicinal, soup, fodder and mulch cover.
\end{abstract}

Key words: Weed Flora, Ethnobotany, Families, Uses, Maiduguri.

\section{INTRODUCTION}

Ethnobotany is the study of the relationship between plants and people Dhanam and Elayaraj (2014). Quite a number of plants considered as weeds in modern sciences have significant value. In ethnobotany, knowledge about local weeds is more important than local knowledge of insectsor plant diseases; some weeds are used as fodder,medicine, even toys, but others are just weeds Bentley et al., 2005. Weeds have genetic and phenotypic ability Dhanam and Elayaraj (2014); such characters enable them to pass through successfully in adverse habitats. They easily invade crop fields which are favourite habitat for their fast growth (Randall 1996; Frohlich et al., 2000; Hassan and Marwat 2001). The presence of weeds in the fields and their impact on the crop production and environment has been well documented. Sher et al., (2011) stated that the unwanted weeds are responsible for the competitive and allelopathic behavior and rendering habitats to harmful organisms. Ethnobotany can help elicit demand for research in aculturally and ecologically sensitive way.Understanding the importance of weeds is useful for taxonomists, agriculturists and scientists involved in the management of weeds.weeds easily invade crop fields which are favourite grounds for their quick growth. The presence of weeds in the fields and their impact on the crop production and environment has been well documented (Randall 1996; Frohlich et al., 2000; Hassan and Marwat 2001). There are numerous benefits of weeds as reported by Soladoye et al., (2010) such as economic and medicinal values, they protect the soil against erosion and add organic matter to the soil. Weeds also provide shelter for beneficial insects and provide nectar for bees, Some weeds serve as raw material for conventional medicine, while others have been used locally for decades for several traditional medicinal purposes ranging from simple laxative to cure dysentery (Hill and Ramsay, 1977; Soladoye et al., 2006).

This study was carried out to ascertain the species of weeds at the experimental farm as this will enable researchers to be aware of the species of plants, their nomenclature and some vital information about the weeds.

$>$ This paper study is to ascertain the species of weeds at Ramat Polytechnic Teaching and Research Farm, Maiduguri Borno State Nigeria.

This paper is to identify the weeds flora and ethnobotanic benefits of these weeds. 


\section{METHODOLOGY}

The study is conducted at the Ramat Polytechnic Teaching and Research farm $\left(40000 \mathrm{~m}^{2}\right)$, in Maiduguri Borno state, between June and July 2019 (at the establishment of rainfall when almost all weeds must have emerged). The ecological information (in the two months of June and July) from the area average tempereature is $36.4^{0} \mathrm{C}-33.2^{\circ} \mathrm{C}$, average rainfall of $73.8 \mathrm{~mm}-147.1 \mathrm{mmin}$ the Sudan savanna regionThe area being a research farm is characterized by numerous annual and perennial vegetation; among the tree crops are Acacia species, neem Azadriratcha indica. Wet and dry season crops such as maize Zea mays, wheat Triticum aestivum, Amaranthus Amaranthus spp, Lettuce Lactuca sativa, Carrot Daucus carota, Onion Allium cepa, Cabbage Brassica oleracea, Sorrel Hibiscus sabdariffa, Okro Hibiscus esculentusTomato Lycopersicon esculentus. Weed samples were collected from $\mathrm{I} \mathrm{m}^{2}$ quadrant around the farm including climbing weeds on wire fence and trees. These weed sample were identified with the help of indigenous farmers, weed identification manual and elders around the community who have the knowledge about traditional medicine and are so familiar with these weeds.

\section{RESULTS AND DISCUSSIONS}

Table 1. Weed Flora of Ramat Polytechnic Teaching and Research Farm, Maiduguri Borno State

\begin{tabular}{|c|c|c|c|c|}
\hline $\mathbf{S} / \mathbf{N}$ & Weed Scientific name & Family & Common name & Lifecycle \\
\hline $\begin{array}{l}1 \\
2 \\
\end{array}$ & $\begin{array}{l}\text { Amaranthus retroflexus } \mathrm{L} . \\
\text { Amaranthus spinosis } \mathrm{L} .\end{array}$ & Amaranthaceae & $\begin{array}{l}\text { Smooth pig weed } \\
\text { Thorn pig weed }\end{array}$ & Annual \\
\hline $\begin{array}{l}3 \\
4\end{array}$ & $\begin{array}{l}\text { Leptadenia hastata (Pers) } \\
\text { Calotropis procera (Aiton) W. T. }\end{array}$ & Apocynaceae & $\begin{array}{l}\text { Akamongot } \\
\text { Sodom apple }\end{array}$ & Annual \\
\hline 5 & Cleome gynandra L. & Cleomaceae & Stink weed & Annual \\
\hline $\begin{array}{l}6 \\
7\end{array}$ & $\begin{array}{l}\text { Commelina benghalensis } \quad \text { Schult. } \\
\text { Commelina erecta linn. }\end{array}$ & Commilanaceae & $\begin{array}{l}\text { Climbing day flower } \\
\text { Wandering jew }\end{array}$ & Annual/perennial \\
\hline 8 & Ipomoea eriocarpa $\mathrm{R} . \mathrm{Br}$. & Convulvulaceae & Tiny morning glory & Annual \\
\hline 9 & Momordica balsamina L. & Curcubitaceae & Balsam apple & Annual \\
\hline 10 & Cyperus rotundus $\mathrm{L}$. & Cyperaceae & Nutsedge & Perennial \\
\hline 11 & Euphorbia hirta linn. & Euphorbiaceae & Asthma plant & Annual \\
\hline $\begin{array}{l}12 \\
13 \\
14 \\
15 \\
16 \\
17 \\
18 \\
19\end{array}$ & $\begin{array}{l}\text { Crotalaria mucronata } \mathrm{L} . \\
\text { Crotalaria medicaginea } \mathrm{L} . \\
\text { Senna tora } \mathrm{L} . \\
\text { Senna occidentalis } \mathrm{L} . \\
\text { Tephrosia purpurea (L.) Pers.) } \\
\text { Swartzia madagascariensisL. } \\
\text { Faidherbia albida A. Cahev. } \\
\text { Acacia nilotica }\end{array}$ & Fabaceae & $\begin{array}{l}\text { Rattle pod } \\
\text { Rattle pod } \\
\text { Sickle senna } \\
\text { Coffee senna } \\
\text { Wild indigo } \\
\text { Snake bean } \\
\text { Acacia } \\
\text { Gum Arabic }\end{array}$ & Anuual/perennial \\
\hline 20 & Ocimum gratissimum L. & Lamiaceae & African basil & Annual \\
\hline 21 & Englerina gabonensis (Engl.) Balle & Loranthaceae & Mistletoe & Perennial \\
\hline $\begin{array}{l}22 \\
23 \\
24\end{array}$ & $\begin{array}{l}\text { Waltheria americana } \mathrm{L} . \\
\text { Hibiscus asper } \mathrm{L} . \\
\text { Sida cordifolia } \mathrm{L} .\end{array}$ & Malvacea & $\begin{array}{l}\text { Sleepy morning } \\
\text { wild sorrel } \\
\text { Flannel weed }\end{array}$ & $\begin{array}{l}\text { Annual } \\
\text { annual } \\
\text { annual }\end{array}$ \\
\hline 35 & Azadirachta indica $\mathrm{L}$ & Meliaceae & Neem & Perennial \\
\hline 26 & Boerhavia erecta $\mathrm{L}$. & Nyctaginaceae & Spiderling & Annual/perennial \\
\hline $\begin{array}{l}27 \\
28 \\
29 \\
30\end{array}$ & $\begin{array}{l}\text { Eragrotis tenella (A. Rich) Hoschst. Ex Steud. } \\
\text { Eragrotis tremula Hochst. } \\
\text { Cynodon dactylon (L.) Pers. } \\
\text { Cenchrus biflorus (Roxb.) }\end{array}$ & Poaceae & $\begin{array}{l}\text { Japanese love grass } \\
\text { Cane grass } \\
\text { Bermuda grass } \\
\text { Indian sandbur }\end{array}$ & $\begin{array}{l}\text { Annual } \\
\text { Annual } \\
\text { Perennial } \\
\text { Annual }\end{array}$ \\
\hline $\begin{array}{l}31 \\
32\end{array}$ & $\begin{array}{l}\text { Mitracarpus hirtus (L.) DC } \\
\text { Borreria stadchydea (DC) Hutch \& Dalziel }\end{array}$ & Rubiaceae & Girdle pod & \\
\hline 33 & Corchorus olitorius L. & Tiliaceae & White jute & Annual \\
\hline 34 & Tribulus terrestris $\mathrm{L}$. & Zygophyllaceae & Devils thorn & Annual \\
\hline
\end{tabular}

Weed flora collected and identified at the experimental farm is presented on Table 1. The weeds were identified with the help of local farmers around and later translated to its various common name, scientific names and families with help of the manual Hausa plant names by Roger Blench (2007). Further clarification and confirmation was done through Google search . Twenty six (26) of the weeds were annuals while the remaining eight (8) are perennials giving a total of thirty four (34) weed species belonging to eighteen (18) families. Two tree species (Acacia and Neem) were included, this is because the seeds of these trees were scattered around and germinate to constitute as the weeds due to the presence of their seedlings. 
Table 2. Weed Flora of Ramat Polytechnic Teaching and Research Farm Maiduguri, Borno State, on Frequency of Occurrence.

\begin{tabular}{|c|c|c|}
\hline $\mathbf{S} / \mathbf{N}$ & Weed Scientific name & $\begin{array}{l}\text { Frequency } \\
\text { occurrence }\end{array}$ \\
\hline $\begin{array}{l}1 \\
2\end{array}$ & $\begin{array}{l}\text { Amaranthus retroflexus } \mathrm{L} . \\
\text { Amaranthus spinosis } \mathrm{L} .\end{array}$ & + \\
\hline 3 & Leptadenia hastata (Pers) & ++ \\
\hline 4 & Calotropis procera (Aiton) W. T. & ++ \\
\hline 5 & Cleome gynandra L. & +++ \\
\hline $\begin{array}{l}6 \\
7\end{array}$ & $\begin{array}{l}\text { Commelina benghalensis Schult. F. } \\
\text { Commelina erecta linn. }\end{array}$ & $\begin{array}{l}+++ \\
+\end{array}$ \\
\hline 8 & Ipomoea eriocarpa $\mathrm{R}$. Br. & ++ \\
\hline 9 & Momordica balsamina L. & ++ \\
\hline 10 & Cyperus rotundus $\mathrm{L}$. & ++ \\
\hline 11 & Euphorbia hirta linn. & +++ \\
\hline $\begin{array}{l}12 \\
13 \\
14 \\
15 \\
16 \\
17 \\
18 \\
19\end{array}$ & $\begin{array}{l}\text { Crotalaria mucronata } \mathrm{L} . \\
\text { Crotalaria medicaginea L. } \\
\text { Senna tora L. } \\
\text { Senna occidentalis L. } \\
\text { Tephrosia purpurea (L.) Pers.) } \\
\text { Swartzia madagascariensis L. } \\
\text { Faidherbia albida A. Cahev. } \\
\text { Acacia nilotica }\end{array}$ & $\begin{array}{l}++ \\
+ \\
+ \\
+++ \\
+ \\
+ \\
++ \\
++\end{array}$ \\
\hline 20 & Ocimum gratissimum L. & + \\
\hline 21 & Englerina gabonensis (Engl.) Balle & + \\
\hline $\begin{array}{l}22 \\
23 \\
24\end{array}$ & $\begin{array}{l}\text { Waltheria americana } \mathrm{L} . \\
\text { Hibiscus asper } \mathrm{L} . \\
\text { Sida cordifolia } \mathrm{L} .\end{array}$ & $\begin{array}{l}+ \\
+ \\
+\end{array}$ \\
\hline 35 & Azadirachta indica $\mathrm{L}$. & ++ \\
\hline 26 & Boerhavia erecta $\mathrm{L}$. & +++ \\
\hline $\begin{array}{l}27 \\
28 \\
29 \\
30\end{array}$ & $\begin{array}{l}\text { Eragrotis tenella (A. Rich) Hoschst. Ex Steud. } \\
\text { Eragrotis tremula Hochst. } \\
\text { Cynodon dactylon (L.) Pers. } \\
\text { Cenchrus biflorus (Roxb.) }\end{array}$ & $\begin{array}{l}+++ \\
+ \\
++ \\
+++\end{array}$ \\
\hline $\begin{array}{l}31 \\
32\end{array}$ & $\begin{array}{l}\text { Mitracarpus hirtus (L.) DC } \\
\text { Borreria stadchydea (DC) Hutch \&Dalziel }\end{array}$ & $\begin{array}{l}++ \\
++\end{array}$ \\
\hline 33 & Corchorus olitorius L. & +++ \\
\hline 34 & Tribulus terrestris L. & +++ \\
\hline
\end{tabular}

KEY $+50 \%$ and below low

$++51 \%-70 \%$ moderate

$+++70 \%-100 \%$ high

Table 2 shows the frequency of occurrence on the weed flora at the research farm. The frequency is categorized into three groups viz; $50 \%$ and below shows the occurrence of the weed in 20 quadrants and less. $51 \%-70 \%$ shows weed occurrence in 21 quadrants to 30 quadrants and $71 \%-100 \%$ shows the occurrence of the weed in all the sampled quadrants (40). In some special cases where parasitic weeds were identified, total number of trees in the whole farm were counted and parasitic weeds identified to give the frequency. This finding is in line with the work of Dhanam and Elayaraj (2014) who reported similar observation on the frequency occurrence of weed.

Table 3. Weed Flora of Ramat Polytechnic Teaching and Research Farm Maiduguri, Borno State, on Percentage of Weed Families

\begin{tabular}{|l|l|l|l|}
\hline S/N & Weed Scientific name & Family & $\begin{array}{l}\text { Percentage } \\
\text { Total 100\% }\end{array}$ \\
\hline 1 & Amaranthus retroflexus L. & Amaranthaceae & 5.88 \\
2 & Amaranthus spinosis L. & & \\
\hline 3 & Leptadenia hastata (Pers) & Apocynaceae & 5.88 \\
4 & Calotropis procera (Aiton) W. T. & &
\end{tabular}


International Journal of Advances in Scientific Research and Engineering (ijasre),Vol 5 (7), July-2019

\begin{tabular}{|c|c|c|c|}
\hline 5 & Cleome gynandra $\mathrm{L}$. & Cleomaceae & 2.94 \\
\hline $\begin{array}{l}6 \\
7\end{array}$ & $\begin{array}{l}\text { Commelina benghalensis Schult. F. } \\
\text { Commelina erecta linn. }\end{array}$ & Commilanaceae & 5.88 \\
\hline 8 & Ipomoea eriocarpa $\mathrm{R} . \mathrm{Br}$. & Convulvulaceae & 2.94 \\
\hline 9 & Momordica balsamina L. & Curcubitaceae & 2.94 \\
\hline 10 & Cyperusrotundus L. & Cyperaceae & 2.94 \\
\hline 11 & Euphorbia hirta linn. & Euphorbiaceae & 2.94 \\
\hline $\begin{array}{l}12 \\
13 \\
14 \\
15 \\
16 \\
17 \\
18 \\
19\end{array}$ & $\begin{array}{l}\text { Crotalaria mucronata } \mathrm{L} . \\
\text { Crotalaria medicaginea } \mathrm{L} . \\
\text { Senna tora } \mathrm{L} . \\
\text { Senna occidentalis } \mathrm{L} . \\
\text { Tephrosia purpurea }(\mathrm{L} .) \text { Pers.) } \\
\text { Swartzia madagascariensis } \mathrm{L} . \\
\text { Faidherbia albida A. Cahev. } \\
\text { Acacia nilotica }\end{array}$ & Fabaceae & 23.53 \\
\hline 20 & Ocimum gratissimum $\mathrm{L}$. & Lamiaceae & 2.94 \\
\hline 21 & Englerina gabonensis (Engl.) Balle & Loranthaceae & 2.94 \\
\hline $\begin{array}{l}22 \\
23 \\
24\end{array}$ & $\begin{array}{l}\text { Waltheria americana } \mathrm{L} . \\
\text { Hibiscus asper } \mathrm{L} . \\
\text { Sida cordifolia } \mathrm{L} .\end{array}$ & Malvacea & 8.82 \\
\hline 35 & Azadirachta indica $\mathrm{L}$. & Meliaceae & 2.94 \\
\hline 26 & Boerhavia erecta $\mathrm{L}$. & Nyctaginaceae & 2.94 \\
\hline $\begin{array}{l}27 \\
28 \\
29 \\
30\end{array}$ & $\begin{array}{l}\text { Eragrotis tenella (A. Rich) Hoschst. Ex Steud. } \\
\text { Eragrotis tremula } \text { Hochst. } \\
\text { Cynodon dactylon (L.) Pers. } \\
\text { Cenchrus biflorus (Roxb.) }\end{array}$ & Poaceae & 11.76 \\
\hline $\begin{array}{l}31 \\
32\end{array}$ & $\begin{array}{l}\text { Mitracarpus hirtus (L.) DC } \\
\text { Borreria stadchydea }(\text { DC) Hutch \& Dalziel }\end{array}$ & Rubiaceae & 5.88 \\
\hline 33 & Corchorus olitorius $\mathrm{L}$. & Tiliaceae & 2.94 \\
\hline 34 & Tribulus terrestris $\mathrm{L}$. & Zygophyllaceae & 2.94 \\
\hline
\end{tabular}

A total of thirty four (34) weed species belonging to eighteen (18) families were identified as constituting the weed flora of the research site. Fabaceae has the largest family with a number of eight (8) weed species followed by the Poaceaefamily with a number of four (4) weed species.Malvaceae family having (3) weed species. All other families constituted the remaining species. This is better illustrated on chart as presented on Fig 1. The prevalence of the (3) three major families was in tandem with the families of the major weeds of the world (Akobundu, 1987).

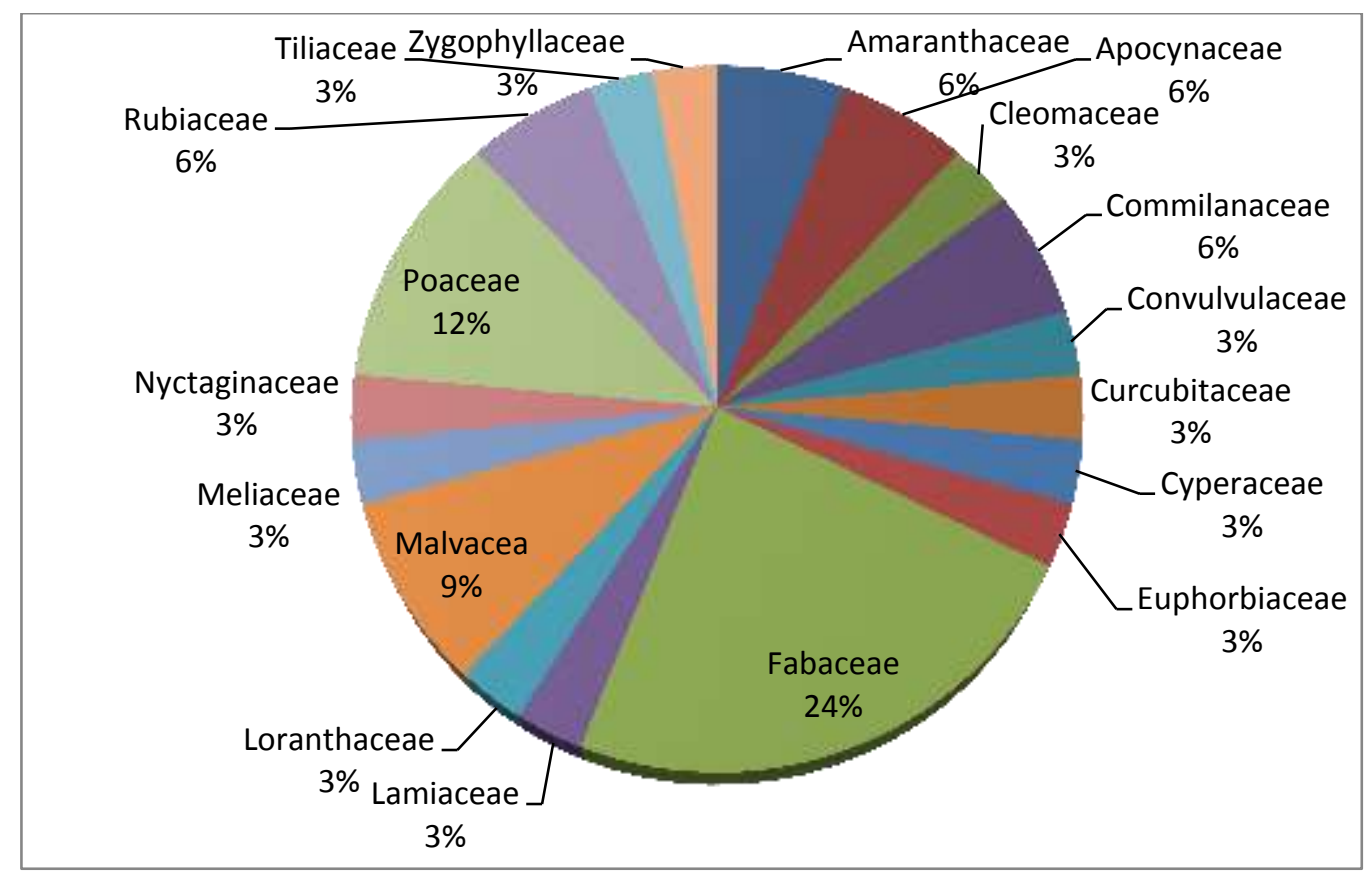

Fig 1. Weed Flora of Ramat Polytechnic Teaching and Research Farm Maiduguri, Borno State, on Percentage of Weed Families. 
Table 4. Weed Flora of Ramat Polytechnic Teaching and Research Farm Maiduguri, Borno State, with Medicinal and other Uses

\begin{tabular}{|c|c|c|}
\hline $\mathbf{S} / \mathbf{N}$ & Weed Scientificname & $\begin{array}{l}\text { Uses; } \\
\text { Medicinal/others * }\end{array}$ \\
\hline $\begin{array}{l}1 \\
2\end{array}$ & $\begin{array}{l}\text { Amaranthus retroflexus L. } \\
\text { Amaranthus spinosis } \mathrm{L} .\end{array}$ & $\begin{array}{l}\text { Fodder } \\
-\end{array}$ \\
\hline $\begin{array}{l}3 \\
4\end{array}$ & $\begin{array}{l}\text { Leptadenia hastata (Pers) } \\
\text { Calotropis procera (Aiton) W. T. }\end{array}$ & $\begin{array}{l}\text { Pot herb, } \\
\text { Medicinal ; Leaves } \\
\text { and sap }\end{array}$ \\
\hline 5 & Cleome gynandra $\mathrm{L}$. & Soup \\
\hline $\begin{array}{l}6 \\
7\end{array}$ & $\begin{array}{l}\text { Commelina benghalensis Schult. F. } \\
\text { Commelina erecta linn. }\end{array}$ & $\begin{array}{l}\text { Fodder } \\
\text { Medicinal }\end{array}$ \\
\hline 8 & Ipomoea eriocarpa $\mathrm{R} . \mathrm{Br}$. & Fodder \\
\hline 9 & Momordica balsamina L. & $\begin{array}{l}\text { Soup, seeds are } \\
\text { medicinal }\end{array}$ \\
\hline 10 & Cyperus rotundus L. & - \\
\hline 11 & Euphorbia hirta linn. & Medicinal \\
\hline $\begin{array}{l}12 \\
13 \\
14 \\
15 \\
16 \\
17 \\
18 \\
19\end{array}$ & $\begin{array}{l}\text { Crotalaria mucronata } \mathrm{L} . \\
\text { Crotalaria medicaginea } \mathrm{L} . \\
\text { Senna tora L. } \\
\text { Senna occidentalis L. } \\
\text { Tephrosia purpurea (L.) Pers.) } \\
\text { Swartzia madagascariensis L. } \\
\text { Faidherbia albida A. Cahev. } \\
\text { Acacia nilotica L. }\end{array}$ & $\begin{array}{l}\text { Highly medicinal } \\
\text { Highly medicinal } \\
\text { Soup } \\
\text { Soup } \\
\text { Highly medicinal } \\
\text { Medicinal } \\
\text { Fodder } \\
\text { Fodder, gum }\end{array}$ \\
\hline 20 & Ocimum gratissimum L. & Medicinal \\
\hline 21 & Englerina gabonensis (Engl.) Balle & - \\
\hline $\begin{array}{l}22 \\
23 \\
24\end{array}$ & $\begin{array}{l}\text { Waltheria americana } \mathrm{L} . \\
\text { Hibiscus asper } \mathrm{L} . \\
\text { Sida cordifolia } \mathrm{L} .\end{array}$ & $\begin{array}{l}\text { Medicinal } \\
\text { Soup } \\
\text { Medicinal }\end{array}$ \\
\hline 35 & Azadirachta indica $\mathrm{L}$. & Medicinal \\
\hline 26 & Boerhavia erecta $\mathrm{L}$. & - \\
\hline $\begin{array}{l}27 \\
28 \\
29 \\
30\end{array}$ & $\begin{array}{l}\text { Eragrotis tenella (A. Rich) Hoschst. Ex Steud. } \\
\text { Eragrotis tremula Hochst. } \\
\text { Cynodon dactylon (L.) Pers. } \\
\text { Cenchrus biflorus (Roxb.) }\end{array}$ & $\begin{array}{l}\text { Mulch } \\
\text { Mulch } \\
\text { Fodder } \\
\text { - }\end{array}$ \\
\hline $\begin{array}{l}31 \\
32\end{array}$ & $\begin{array}{l}\text { Mitracarpus hirtus (L.) DC } \\
\text { Borreria stadchydea (DC) Hutch \&Dalziel }\end{array}$ & $\begin{array}{l}\text { Sap medicinal } \\
\text { Medicinal }\end{array}$ \\
\hline 33 & Corchorus olitorius L. & Soup \\
\hline 34 & Tribulus terrestris L. & - \\
\hline
\end{tabular}

*information given not on scientific findings but through oral interaction with the indigenous people around

Table 4 presents the weed flora of the research farm with their medicinal and other uses, most of the species are medicinal through their different parts (sap, leaves, stem, seeds, roots) fresh or dried, prepared with some other ingredients. Among these are the Crotalaria species and Senna occidentalis which are highly medicinal (as anti fever) especially in small children; as stated by Ogunkunle and Ladejobi (2006)local usage of the plant for these purposes and the presence of anthraquinones in its leaf extracts. Many out of the sampled weeds are used as pot herbs or soups according to different traditions. Other uses include fodder, mulching material and soil cover this is in line with the findings of many researchers on ethnobotany of weeds (Solodoye et al., 2010; Muhammad et al., 2018; )

\section{CONCLUSION}

In conclusion, weed flora of Ramat Polytechnic Teaching and Research Farm, Maiduguri Borno state shows that 38 weed species were identified belonging to 18 different families out of which mostly, twenty six (26) are annuals the remaining (8) are perennials. The distribution of these weeds further shows that Cleome gynandra L., Commelina benghalensis Schult. F., Euphorbia hirta linn., Crotalaria mucronata L., Senna occidentalis L., Boerhavia erecta L., Eragrotis tenella (A. Rich) Hoschst. Ex Steud.,Cenchru sbiflorus (Roxb.)., Corchorus olitorius L. and Tribulus terrestris L. have high (70\%) occurrence at the farm. The dominant plant families are the Fabaceae, Poaceae and Malvaceae. Most of the samples weeds have uses ranging from medicinal, soup, fodder and mulch cover. 


\section{RECOMMENDATION}

Further studies should be conducted about the allelopathic effects of these weeds, their benefit as all plants are important in one way or the other.

\section{REFERENCES}

1. Akobundu, I.O. (1987). Weed Science in the Tropics: Principles and practices. John Wiley \& Sons, Chichester,pp. 522.

2. Dhanam S. and Elayara B. (2014) Ethnomedicinal aspects of some weeds from paddy fields of Villupuram district in Tamil Nadu, India International Letters of Natural Sciences 14 (2014) 1-10 ISSN 2300-9675

3. Frohlich J., S.V. Fower, A. Gianotti, R. Hill, E. Kilgore, L. Morin, C. Winks, (2000) Xth International Symp. On Biological Control of Weeds, 4-14 July 1999, Bozeman, Montana USA, P. 51-57.

4. Hassan G., K. B. Marwat, (2001) Integrated weed management in agricultural crop. National Workshop on Technologies for Sustainable,.

5. Hill, B.S. and Ramsay, J. (1977). Weeds as Indicators of Soil Conditions. Ecological Agricultural Projects Publication 67.

6. Jeffery W. B, Morag W., Silvio N. and Salomo P. (2005). Even Useful Weeds are Pests: Ethnobotany in the Bolivian Andes International Journal of Pest Management, 51(3): 189 - 207

7. Khan M. N., Razzaq A., Hadi F., Khan N., Basit A., Jan F., Khan N. (2018). Ethnobotanical profile of weed flora of district Charsadda, Khyber Pakhtunkhwa. RADS J. Biol. Res. Appl. Sci.; 9(1): 14-23

8. Ogunkunle A.T.J. and Ladejobi T. A. (2006) Ethnobotanical and phytochemical studies on some species of Sennain Nigeria African Journal of Biotechnology Vol. 5 (21), pp. 2020-2023

9. Randall, J. M. (1996).Weed Tech., 10 370-381.

10. Roger B., (2007). Hausa Names for Plants and Trees. $2^{\text {nd }}$ edition. http://www.rogerblench.info/RBOP.htm

11. Sher Z. F., Hussain L., Badshah M., Wahab M. (2011) Floristic composition, communities and ecological characteristics of weeds of wheat fields of Lahore, District Swabi, Pakistan. Pak J Bot.; 43(6): 2817-20.

12. Soladoye M.O., Osipitan A.A., Sonibare M.A., ,Chukwuma E.C. (2010) From 'Vagabonds' to Ethnobotanical Relevance: Weeds of the Campus Sites of OlabisiOnabanjo University, Ago-Iwoye, Nigeria. Ethnobotanical Leaflets 14: 546-58.

13. Soladoye, M.O., Yakubu, F.A., Kola-Oladiji, K., Alabi,, D.A. and Agbomeji, Y.O. (2006). The Collection, Conservation and Cultivation of Local Medicinal Plants for Natural Medicine Production. The Seminar/Workshop and Exhibition of natural Medicine products. Organized by Nigeria Traditional Medical Association, Ijebu-North in collaboration with Faculty of Science, OlabisiOnabanjo University, Ago-Iwoye held 19th-23rd September, 2006. 